\title{
FIXED POINTS OF SYMMETRIC PRODUCT MAPPINGS
}

CHARLES N. MAXWELL

1. Introduction. Let $X$ be a topological space and $X^{n}$ the $n$th cartesian product in the usual topology. Let $G$ be any group of permutations of the letters $[1, \cdots, n]$. Then $G$ can be considered as a group of homeomorphisms on $X^{n}$ by defining, for $g \in G$ and $\left(x_{1}, \cdots, x_{n}\right) \in X^{n}, g\left(x_{1}, \cdots, x_{n}\right)=\left(x_{g(1)}, \cdots, x_{g(n)}\right)$. The orbit space under the group (in the identification topology) will be denoted by $X^{n} / G$ and called a $G$-product of $X$. Let $\eta: X \rightarrow X^{n} / G$ be the identification map. ${ }^{1}$

We will consider continuous functions $f: X \rightarrow X^{n} / G$ and will say that an element $x \in X$ is fixed under $f$ if $f(x)$ has $x$ as one of its coordinates. The purpose of this paper is to associate to each $G$-product map a Lefschetz number (an integer depending upon the homotopy class of the function) with the property that if this number is not zero, then the function has a fixed point [2]. The Lefschetz number will be defined only in case $X$ is a polyhedron. An application will be given regarding spaces with a finite group of operators.

2. $G$-products and a special homology homomorphism. Let the $i$ th projection of $X^{n}$ on to $X$ be denoted by $\pi_{i}$. It is clear that the group and the projections are interrelated by $\pi_{i} g(z)=\pi_{g(i)} z$ for $z \in X^{n}$ and $g \in G$.

In case $X$ is metric, then using the usual euclidean metric on $X^{n}$, $G$ becomes a group of isometries on $X^{n}$. A metric may be introduced in $X^{n} / G$ by defining

$$
d\left(\eta(z), \eta\left(z^{\prime}\right)\right)=\inf \left\{d\left(z, g z^{\prime}\right) \mid g \in G\right\}
$$

where $z, z^{\prime} \in X^{n}$. It is convenient also to introduce a metric-like function on $X \times\left(X^{n} / G\right)$ defined by

$$
\omega(x, \eta(z))=\inf \left\{d\left(x, \pi_{i}(z)\right) \mid i=1, \cdots, n\right\}
$$

where $x \in X$ and $z \in X^{n}$. Then $\omega$ is continuous and satisfies the inequality:

Received by the editors August 3, 1956 and, in revised form, November 21, 1956 .

1 Part of this paper is drawn from the author's dissertation at the University of Illinois. The author wishes to express his appreciation to D. G. Bourgin for his valuable guidance in the preparation of the dissertation. 


$$
\omega(x, y) \leqq \omega\left(x, y^{\prime}\right)+d\left(y^{\prime}, y\right)
$$

for any $x \in X$ and $y, y^{\prime} \in X^{n} / G$.

Now let $X=|K|$ be a polyhedron. Denote the set of vertices of $K$ by $V$ and the set simplexes of $K$ by $S$. Assume that $K$ is an ordered complex, that is to say that a partial ordering $\leqq$ is defined on $V$ which is a linear ordering on any subset of $V$ in $S$. A triangulation $K^{n}$ of $X^{n}$ may be obtained as follows [1, p. 67]: The set of vertices of $K^{n}$ is $V^{n}$. Let $\pi_{i}: V^{n} \rightarrow V$ be the $i$ th projection. For $w$, $w^{\prime} \in V^{n}$, define $w \leqq w^{\prime}$ if $\pi_{i} w \leqq \pi_{i} w^{\prime}$ for all $i=1, \cdots, n$. A subset $t=\left(w_{0}, \cdots, w_{p}\right)$ of $V^{n}$ is a simplex of $K^{n}$ if it is linearly ordered and the vertices $\pi_{i} w_{0}, \cdots, \pi_{i} w_{p}$ span a simplex of $K$ for $i=1, \cdots, n$ (the vertices $\pi_{i} w_{0}, \cdots, \pi_{i} w_{p}$ need not be distinct). It follows from the definition of $K^{n}$ that the projections are simplicial and that $G$ is a group of order preserving functions on $K^{n}$. Since each $g \in G$ is order preserving on $V^{n}$, we have that if $(w, g w)$ is a simplex of $K^{n}$, then $w \leqq g w \leqq g^{2} w$ $\leqq \ldots \leqq g^{k} w=w$ for some integer $k$, and hence $g w=w$.

Let $S d\left(K^{n}\right)$ denote the first barycentric subdivision of $K^{n}$ (the set $B$ of vertices of $S d\left(K^{n}\right)$ consists in all barycenters $b_{t}$ of simplexes $t$ of $\left.K^{n}\right)$. The group $G$ operates on $S d\left(K^{n}\right)$ by $g b_{t}=b_{o t}$ and the simplicial map $\phi: S d\left(K^{n}\right) \rightarrow K^{n}$ (which associates to $b_{t}$ the least vertex of $t$ ) commutes with each $g \in G$. Furthermore, if $\left(b_{t}, b_{t^{\prime}}\right)$ and $\left(b_{t}, g b_{t^{\prime}}\right)$ are both simplexes of $S d\left(K^{n}\right)$, then $g b_{t}=b_{t}$. For if $\left(b_{t}, b_{t^{\prime}}\right)$ and $\left(g^{-1} b_{t}, b_{t^{\prime}}\right)$ are both simplexes of $S d\left(K^{n}\right)$ then $t$ and $g^{-1}(t)$ are both faces of $t^{\prime}$. If we let $w$ denote any vertex of $t$, then $\left(w, g^{-1} w\right)$ is a simplex of $K^{n}$. By a previous argument, $w=g w$. Hence $t=g t$ and $g b_{t}=b_{t}$.

A triangulation $K(n, G)$ for $X^{n} / G$ can now be defined. The set $A$ of vertices of $K(n, G)$ is the set of equivalence class of elements of $B$ under $G$. A subset $\left(a_{0}, \cdots, a_{p}\right)$ of $A$ form a simplex of $K(n, G)$ if there exists $b_{i} \in a_{i}$ so that $\left(b_{0}, \cdots, b_{p}\right)$ is a simplex of $S d\left(K^{n}\right)$. If another choice is made, say $b_{i}^{\prime} \in a_{i}$ so that $\left(b_{0}^{\prime}, \cdots, b_{p}^{\prime}\right)$ is a simplex of $S d\left(K^{n}\right)$, then $b_{i}^{\prime}=g_{i} b_{i}$ for some $g_{i} \in G, i=0 \cdots p$. For any $i$ we have $\left(b_{i}, b_{p}\right)$ and $\left(g_{i} b_{i}, g_{p} b_{p}\right)$ simplexes of $S d\left(K^{n}\right)$, and therefore $\left(b_{i}, b_{p}\right)$ and $\left(b_{i}, g_{i}{ }^{-1} g_{p} b_{p}\right)$ are simplexes of $S d\left(K^{n}\right)$. By a previous argument, we have $g_{i}^{-1} g_{p} b_{i}=b_{i}$, and hence $g_{p} b_{i}=g_{i} b_{i}$. Therefore

$$
\begin{aligned}
\left(b_{0}{ }^{\prime}, \cdots, b_{p}{ }^{\prime}\right) & =\left(g_{0} b_{0}, \cdots, g_{p} b_{p}\right)=\left(g_{p} b_{0}, \cdots, g_{p} b_{p}\right) \\
& =g_{p}\left(b_{0}, \cdots, b_{p}\right) .
\end{aligned}
$$

The $p$-simplexes of $K(n, G)$ are therefore in one-to-one correspondence with the equivalence classes of $p$-simplexes of $S d\left(K^{n}\right)$.

Consider now the integral chain groups defined on oriented simplexes of these complexes: 


$$
\sum_{i=1}^{n} \begin{array}{cc}
C_{p}\left(K^{n}\right) \stackrel{\phi \sharp}{\leftarrow} & C_{p}\left(S d\left(K^{n}\right)\right) \\
C_{p}(K) & C_{p}(K(n, G))
\end{array}
$$

We wish to complete the rectangle with a homomorphism $\mu$ : $C_{p}(K(n, G)) \rightarrow C_{p}(K)$ so that the diagram commutes ( $\sum_{i=1}^{n} \pi_{i \neq}$ is the sum of the projection chain maps). Let $t=\left(a_{0}, \cdots, a_{p}\right)$ be a generator of $C_{p}(K(n, G))$. Choose any $\sigma=\left(b_{0}, \cdots, b_{p}\right)$ generator of $C_{p}\left(S d\left(K^{n}\right)\right)$ for which $\eta \#(\sigma)=t$. Then define

$$
\mu(t)=\sum_{i=1}^{n} \pi_{i \sharp \phi}(\sigma) .
$$

The definition is independent of the choice of $\sigma$. For if $\eta \#\left(\sigma^{\prime}\right)=\eta^{\sharp}(\sigma)$ $=t$, then $\sigma^{\prime}=g \sigma$ for some $g \in G$, and

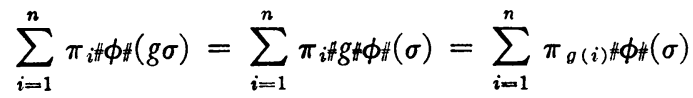

$$
\begin{aligned}
& =\sum_{i=1}^{n} \pi_{i \sharp \sharp} \phi(\sigma) .
\end{aligned}
$$

Also, $\mu$ is a chain map, since $\eta \#, \phi \#$, and $\sum_{i=1}^{n} \pi_{i \#}$ each commute with the boundary operator, and hence $\mu$ induces $\mu_{*}^{d}: H_{p}\left(X^{n} / G\right) \rightarrow H_{p}(X)$.

From the commutativity of the above mapping diagram, we have $\sum_{i=1}^{n} \pi_{i *}=\mu_{*} \eta_{*}$ as mappings from $H_{p}\left(X^{n}\right)$ into $H_{p}(X)$. In general a $G$-product mapping $f: X \rightarrow X^{n} / G$ cannot be factored through $X^{n}$. However, if $f$ can be expressed as a composite $\eta f^{\prime}$ where $f^{\prime}: X \rightarrow X^{n}$, then

$$
\mu_{*} f_{*}=\mu_{*} \eta_{*} f_{*}^{\prime}=\sum_{i=1}^{n} \pi_{i *} f_{*}^{\prime}=\sum_{i=1}^{n} f_{i *}^{\prime}
$$

where $f_{i}^{\prime}$ is the $i$ th component $\pi_{i} f^{\prime}$ of $f^{\prime}$.

3. The topological invariance of $\mu_{*}$. Let $X$ and $Y$ be any two spaces and $F: X \rightarrow Y$ a continuous function. Then $F$ induces a map $\tilde{F}: X^{n} / G$ $\rightarrow Y^{n} / G$ defined as follows: Let $F^{n}: X^{n} \rightarrow Y^{n}$ be given by $F^{n}\left(x_{1}, \cdots, x_{n}\right)$ $=\left(F x_{1}, \cdots, F x_{n}\right)$. Then $F^{n}$ is equivariant with respect to $G$ and hence induces a continuous map $\tilde{F}$ on the quotient spaces. If $F_{1}: X \rightarrow Y$ and $F_{2}: Y \rightarrow Z$, then clearly the function induced on the corresponding $G$ products by the composite $F_{2} F_{1}$ is the composite $\tilde{F}_{2} \tilde{F}_{1}$. Also, if $F_{0}$ and $F_{1}$ are two maps of $X$ into $Y$ which are homotopic, then $\widetilde{F}_{0}$ and $\tilde{F}_{1}$ are homotopic. If $F: X \rightarrow Y$ is a homeomorphism, then $\tilde{F}$ is also. 
Let $X=|K|$ and $Y=|L|$ be polyhedra where $K$ and $L$ are ordered complexes. Then the complexes $K(n, G)$ and $L(n, G)$ are defined as before and depend upon the vertex ordering of $K$ and $L$, respectively. Let $f: K \rightarrow L$ be a simplicial order preserving function. Then $f^{n}$ can be defined on the vertices of $K^{n}$ into the vertices of $L^{n}$ by $f^{n}\left(v_{1} \times \cdots \times v_{n}\right)=\left(f v_{1} \times \cdots \times f v_{n}\right)$ and $f^{n}$ is order preserving on the vertices of $K^{n}$. So, if $w_{0} \leqq \cdots \leqq w_{p}$ is a simply ordered set of vertices of $K^{n}, f^{n} w_{0} \leqq \cdots \leqq f^{n} w_{p}$ is simply ordered. Furthermore, $\bar{\pi}_{i} f^{n}=f \pi_{i}$ for all $i=1, \cdots, n$ where $\pi_{i}$ and $\bar{\pi}_{i}$ are the projections in $K$ and $L$, respectively. Hence, $f^{n}: K^{n} \rightarrow L^{n}$ is a simplicial map and induces $\left(f^{n}\right)^{\prime}: S d\left(K^{n}\right) \rightarrow S d\left(L^{n}\right)$ in the usual fashion. The map $f^{n}$ is equivariant with respect to $G$ and hence $\left(f^{n}\right)^{\prime}$ is also. Therefore, $\left(f^{n}\right)^{\prime}$ induces a simplicial function $\tilde{f}: K(n, G) \rightarrow L(n, G)$, and the continuous function induces on $|K|^{n} / G$ into $|L|^{n} / G$ by $f$ is the same as the simplicial map $\tilde{f}$. If we denote by $\phi: S d\left(K^{n}\right) \rightarrow K^{n}$ and $\phi: S d\left(L^{n}\right) \rightarrow L^{n}$ the order preserving subdivision maps, then we have $f^{n} \phi=\Phi\left(f^{n}\right)^{\prime}$. If $\eta: S d\left(K^{n}\right)$ $\rightarrow K(n, G)$ and $\bar{\eta}: S d\left(L^{n}\right) \rightarrow L(n, G)$ are the identification maps, then $\tilde{f} \eta=\bar{\eta}\left(f^{n}\right)^{\prime}$.

Lemma 1. If $f: K \rightarrow L$ is an order preserving simplicial map, then $f_{*} \mu_{K *}=\mu_{L *} \tilde{f}_{*}$.

Proof. Take $\sigma$ a generator of $C_{p}(K(n, G))$ and let $t$ be a generator of $C_{p}\left(S d\left(K^{n}\right)\right)$ for which $\eta(t)=\sigma$. Then

$$
\begin{aligned}
& f_{t} \mu_{K}(\sigma)=f_{t} \sum_{i=1}^{n} \pi_{i} \phi_{t}(t) \\
& =\sum_{i=1}^{n} \bar{\pi}_{i f f} f_{i \neq}^{n}(t) \\
& =\sum_{i=1}^{n} \bar{\pi}_{i f} \bar{\phi}_{f}\left(f^{n}\right) f^{\prime}(t) \\
& =\mu_{L}\left(\tilde{f}_{*} \sigma\right)
\end{aligned}
$$

since $\bar{\eta}\left(\left(f^{n}\right)^{\prime}(t)\right)=\tilde{f} \eta(t)=\tilde{f}(\sigma)$. Q.E.D.

Leмма 2. If $f: K \rightarrow L$ is any simplicial function (not necessarily order preserving), then $f_{*} \mu_{K *}=\mu_{L *} \tilde{f}_{*}$.

Proof. Let $K^{\prime}$ and $L^{\prime}$ be the barycentric subdivision of $K$ and $L$, respectively. Let $\alpha: K^{\prime} \rightarrow K$ and $\beta: L^{\prime} \rightarrow L$ be the simplicial maps which associate to each barycenter of a simplex the least vertex of the simplex. Then $\alpha$ and $\beta$ are order preserving. Let $f^{\prime}: K^{\prime} \rightarrow L^{\prime}$ be the simplicial map induced by $f$. Then $f^{\prime}$ is order preserving, and we have 


$$
\begin{aligned}
& H_{p}(K) \stackrel{\alpha_{*}}{\longleftarrow} H_{p}\left(K^{\prime}\right) \stackrel{f_{*}^{\prime}}{\longrightarrow} H_{p}\left(L^{\prime}\right) \stackrel{\beta_{*}}{\longrightarrow} H_{p}(L) \\
& \uparrow \mu_{K *} \\
& \mu_{K^{\prime} *} \stackrel{\tilde{\alpha}_{*}}{\longleftarrow} H_{p}\left(K^{\prime}(n, G)\right) \stackrel{\tilde{f}^{\prime}}{\longrightarrow} H_{p}\left(\mu_{L^{\prime} *}(n, G)\right) \stackrel{\tilde{\beta}_{*}}{\longrightarrow} H_{L *} H_{p}(L(n, G)) .
\end{aligned}
$$

By Lemma 1, each rectangle commutes. It is clear that $\alpha_{*}$ is an isomorphism and that $f_{*}=\beta_{*} f_{*}^{\prime} \alpha_{*}^{-1}$. Since $\alpha$ and $\beta$ are homotopic to the identity on $|K|$ and $|L|$, respectively, $\tilde{\alpha}$ and $\tilde{\beta}$ are homotopic to the identity on $|K(n, G)|$ and $|L(n, G)|$. Therefore, $\tilde{\alpha}_{*}$ and $\tilde{\beta}_{*}$ are isomorphisms. Since $f \alpha$ and $\beta f^{\prime}$ are homotopic, we have $\tilde{f} \tilde{\alpha}$ homotopic to $\tilde{\beta} \tilde{f}^{\prime}$. Hence, $\tilde{f}_{*} \tilde{\alpha}_{*}=\widetilde{\beta}_{*} \tilde{f}_{*}^{\prime}$ and $\tilde{f}_{*}=\tilde{\beta}_{*} \tilde{f}_{*}^{\prime} \tilde{\alpha}_{*}^{-1}$. Therefore, $f_{*} \mu_{K *}=\mu_{L *} \tilde{f}_{*}$.

Leмma 3. If $F:|K| \rightarrow|L|$ is any continuous function, then $F_{*} \mu_{K *}$ $=\mu_{L *} \widetilde{F}_{*}$.

Proof. Let $N$ be a barycentric subdivision of $K$, sufficiently fine so that a simplicial map $f: N \rightarrow L$ exists which is a simplicial approximation to $F[2]$. Let $\gamma: N \rightarrow K$ be a simplicial subdivision map. We have the diagram:

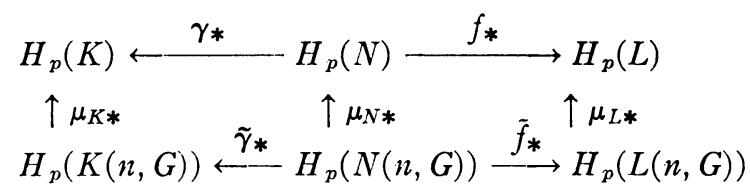

By the previous lemma, each rectangle commutes. In the top row, $\gamma_{*}$ is an isomorphism and $F_{*}=f_{*} \gamma_{*}^{-1}$. Since $\gamma$ is homotopic to the identity on $|K|, \tilde{\gamma}$ is homotopic to the identity on $|K|^{n} / G$ and $\tilde{\gamma}_{*}$ is an isomorphism. Since $F \gamma$ is homotopic to $F$, we have $\tilde{F} \tilde{\gamma}$ homotopic to $\tilde{F}$. Hence $\tilde{F}_{*} \tilde{\gamma}_{*}=\tilde{f}_{*}$ and $\tilde{F}_{*}=\tilde{f}_{*} \tilde{\gamma}_{*}^{-1}$. Therefore, $F_{*} \mu_{K *}=\mu_{L *} \tilde{F}_{*}$.

Now if we take $F$ to be a homeomorphism, we have:

COROLlaRy. The homomorphism $\mu_{*}$ is independent of the triangulation.

4. Main theorem. As before, let $X=|K|$ be a polyhedron and let $f: X \rightarrow X^{n} / G$ be a continuous function. In the remainder of the paper the coefficient group for the homology groups will be the field of rational numbers. Consider the composite homomorphism

$$
H_{p}(X) \stackrel{f_{*}^{p}}{\longrightarrow} H_{p}\left(X^{n} / G\right) \stackrel{\mu_{*}}{\longrightarrow} H_{p}(X)
$$

Since $\mu_{*}^{p} f_{*}^{p}$ is a linear transformation of a finite dimensional vector space into itself, the trace of $\mu_{*}^{p} f_{*}^{p}$ is defined. The Lefschetz number $\mathcal{L}(f)$ is defined to be the number $\sum_{p=0}^{\infty}(-1)^{p}$ trace $\left(\mu_{*}^{p} f_{*}^{p}\right)$. Since $f_{*}^{p}$ 
and $\mu_{*}^{p}$ are independent of the triangulation, $\mathscr{L}(f)$ is also. In case $n=1$, then $\mu_{*}^{p}$ is the identity and $\mathfrak{L}(f)$ reduces to the Lefschetz number as defined in [2].

THEOREM 1. If $X$ is a polyhedron and $f: X \rightarrow X^{n} / G$ where $\mathfrak{L}(f) \neq 0$, then $f$ has a fixed point.

Proof. Assume, by way of contradiction, that $f$ has no fixed points. Then $\omega(x, f(x))>0$ for all $x \in X$. Since $X$ is compact, there exists $\epsilon>0$ such that $\omega(x, f(x))>\epsilon$ for all $x \in X$.

Let a triangulation $K$ of $X$ be chosen so small that mesh $K^{n}<\epsilon / 3$. Then clearly mesh $K<\epsilon / 3$ and mesh $K(n, G)<\epsilon / 3$. By the simplicial approximation theorem, there is a subdivision $K_{1}$ of $K$ and a map $h: K_{1} \rightarrow K(n, G)$ which is a simplicial approximation to $f$. In particular, $d(h(x), f(x))<\epsilon / 3$ for all $x \in X$.

Consider the composite chain map

$$
C_{p}(K) \stackrel{\nu}{\rightarrow} C_{p}\left(K_{1}\right) \stackrel{h_{*}}{\rightarrow} C_{p}(K(n, G)) \stackrel{\mu}{\rightarrow} C_{p}(K)
$$

where $\nu$ denotes the subdivision chain map. Let $t$ denote the settransformation [2] which associates to each open simplex $s$ of $K$ the closed subset $t(s)=\bigcup_{i=1}^{n} \pi_{i} \phi \eta^{-1} h(|s|)$ of $X$. Then it is clear that $t$ is a carrier of the above composite chain map $\mu h_{\sharp \nu}$.

We shall now show that $|s| \cap t(s)=\phi$ for every simplex $s$ of $K$. Assume, by way of contradiction, that $x \in|s| \cap t(s)$ for some $s$ of $K$. Then there exists $z \in X^{n}$ for which $\pi_{i} z=x$ for some $i$ and $z \in \phi \eta^{-1} h(|s|)$. Since $z \in \phi \eta^{-1} h(|s|)$, there exists $z^{\prime} \in \eta^{-1} h(|s|)$ with $\phi\left(z^{\prime}\right)=z$. Hence $d\left(z, z^{\prime}\right)<\epsilon / 3$ in $X^{n}$. Since $z^{\prime} \in \eta^{-1} h(|s|)$, there exists $y \in|s|$ such that $h(y)=\eta\left(z^{\prime}\right)$ and since $x$ and $y \in|s|$, we have $d(x, y)<\epsilon / 3$ in $X$. Therefore $\omega(y, \eta(z))<\epsilon / 3$, since $\pi_{i} z=x$ and $d(x, y)<\epsilon / 3$. But

$$
\begin{aligned}
\omega(y, f(y)) & \leqq \omega(y, \eta(z))+d(\eta(z), f(y)) \\
& \leqq \omega(y, \eta(z))+d\left(\eta(z), \eta\left(z^{\prime}\right)\right)+d(h(y), f(y)) \\
& <\epsilon / 3+\epsilon / 3+\epsilon / 3=\epsilon .
\end{aligned}
$$

This inequality contradicts the choice of $\epsilon$. Therefore $|s| \cap t(s)=\phi$ for all simplexes $s$ of $K$. It follows that the chain mapping $\mu^{p} h_{t \nu^{p}}^{p}$ has, when expressed as a matrix, all zero entries on the diagonal. Therefore the trace of $\mu^{p} h_{i t}^{p} \nu^{p}=0$ for all $p$, and hence $\sum_{p=0}^{\infty}(-1)^{p} \operatorname{trace}\left(\mu^{p} h_{\| \nu^{p}}^{p}\right)$ $=0$. But the alternating sum of the traces of a chain map is equal to the alternating sum of the traces of its induced homology homomorphism [2]. Hence

$$
\sum_{p=0}^{\infty}(-1)^{p} \operatorname{trace}\left(\mu_{*}^{p} h_{*}^{p} \nu_{*}^{p}\right)=\sum_{p=0}^{\infty}(-1)^{p} \operatorname{trace}\left(\mu_{*}^{p} f_{*}^{p}\right)=\mathscr{L}(f)=0
$$


since $\nu_{*}^{p}$ is the identity and $h$ is homotopic to $f$. But this contradicts the hypothesis that $\mathfrak{L}(f) \neq 0$. Therefore $f$ has a fixed point. Q.E.D.

COROllary. If $X$ is an acyclic polyhedron, then every function $f: X \rightarrow X^{n} / G$ has a fixed point.

Proof. Since $X$ is connected, the group $H_{0}(x)$ is one dimensional over the rationals. Let $z_{0}$ be any nonzero element of $H_{0}(X)$. It is straight-forward to check that $\mu_{*}^{0} f_{*}^{0}\left(z_{0}\right)=n z_{0}$. Since $H_{p}(X)=0$ for $p>0$, we have $\mu_{*}^{p} f_{*}^{p}=0$ for $p>0$. Hence $\mathscr{L}(f)=n \neq 0$ and $f$ has a fixed point. Q.E.D.

Let $X$ be a connected polyhedron and let $x_{0}$ be an arbitrary point of $X$. For any integer $k$ where $1 \leqq k \leqq n$, define a map $d_{k}: X \rightarrow X^{n}$ to be the identity on the first $k$ factors and the constant value $x_{0}$ on the remaining $n-k$ factors. Let $\bar{d}_{k}=\eta d_{k}$. Since $X$ is connected, the homotopy classes of $d_{k}$ and $\bar{d}_{k}$ are independent of $x_{0}$. For all $k, \bar{d}_{k}$ leaves every element of $X$ fixed.

CoROLlaRy. If the Euler characteristic $X(X) \neq(k-n) / k$, then every function $f: X \rightarrow X^{n} / G$ homotopic to $\bar{d}_{k}$ has a fixed point.

Proof. Since $f$ is homotopic to $\bar{d}_{k}$, we have $\mathfrak{L}(f)=\mathfrak{L}\left(\bar{d}_{k}\right)=\mathfrak{L}\left(\eta d_{k}\right)$. But $\mathscr{L}\left(\eta d_{k}\right)$ is the sum of the Lefschetz numbers of the components of $d_{k}$. Hence $\mathscr{L}(f)=k \mathfrak{X}(X)+n-k$. The hypothesis implies that $\mathfrak{L}(f) \neq 0$. Therefore $f$ has a fixed point. Q.E.D.

5. Spaces with groups of operators. Coincidences. Let $Y$ be a space and $G$ a group of operators on $Y[1]$. Assume that $G$ is finite and let $g_{1}, \cdots, g_{n}$ be any enumeration of the elements of $G$. Let $Y / G$ be the space of orbits under $G$ in the identification topology and let $\pi: Y \rightarrow Y / G$ be the identification map.

Let $Y^{(n)}=Y^{n} / S_{n}$ be the $n$th symmetric product of $Y$. Then the orbit space $Y / G$ can be embedded in $Y^{(n)}$ as follows: Consider the map $\rho: Y \rightarrow Y^{n}$ whose components are the elements of $G$, that is $\rho(y)$ $=\left(g_{1} y, \cdots, g_{n} y\right)$. For any $g \in G, \rho(g y)=\left(g_{1} g y, \cdots, g_{n} g y\right)$ which is equivalent to $\rho(y)$ in $Y^{n}$ under the symmetric group. Hence $\rho$ induces a single-valued function $\bar{\rho}: Y / G \rightarrow Y^{(n)}$ which can be shown to be a homeomorphism, and the following diagram commutes:

$$
\begin{array}{crr}
Y & \stackrel{\rho}{\rightarrow} Y^{n} \\
\pi \downarrow & & \downarrow \eta \\
Y / G \stackrel{\rho}{\rightarrow} & Y^{(n)}
\end{array}
$$

Now let $f: Y \rightarrow Y / G$ be an arbitrary continuous function. 
ThEOREM 2. If $Y$ is a polyhedron and $\mathfrak{L}(\bar{\rho} f) \neq 0$, then $f$ and $\pi$ have a coincidence.

Proof. If $\mathcal{L}(\bar{\rho} f) \neq 0$, then $\bar{\rho} f$ has a fixed point. Therefore there exists $y \in Y$ which is a coordinate of $\bar{\rho} f(y)$. Let $f(y)=x \in Y / G$ and let $y^{\prime}$ be any element of $Y$ such that $\pi\left(y^{\prime}\right)=x$. Then $\bar{\rho} f(y)=\bar{\rho}(x)$ $=\left(g_{1} y^{\prime}, \cdots, g_{n} y^{\prime}\right)$. Hence $y=g_{i} y^{\prime}$ for some $g_{i}$, and $\pi(y)=\pi\left(y^{\prime}\right)=x$ $=f(y) \cdot$ Q.E.D.

Corollary 1. Let $Y$ and $Y / G$ be polyhedra. If either $Y$ or $Y / G$ is acyclic, then any function $f: Y \rightarrow Y / G$ has a coincidence with $\pi$.

PROOF. In each case $\mathscr{L}(\bar{\rho} f)$ is easily seen to be nonzero. Q.E.D.

CoROLlaRy 2. If $Y$ is a polyhedron and $\sum_{i=1}^{n} \mathscr{L}\left(g_{i}\right) \neq 0$, then every function $f: Y \rightarrow Y / G$ homotopic to $\pi$ has a coincidence with $\pi$.

Proof. If $f$ is homotopic to $\pi$, then $\bar{\rho} f$ is homotopic to $\bar{\rho} \pi$. But $\bar{\rho} \pi=\eta \rho$. Therefore $\mathscr{L}(\bar{\rho} f)=\mathfrak{L}(\eta \rho)=\sum_{i=1}^{n} \mathfrak{L}\left(g_{i}\right)$ since the elements of the group are the components of $\rho$. By hypothesis $\sum_{i=1}^{n} \mathscr{L}\left(g_{i}\right) \neq 0$, so $f$ has a coincidence with $\pi$. Q.E.D.

EXAMPLE. Let $X$ be a $k$-sphere, and let $G$ be any finite group of homeomorphisms on $X$. Then if $k$ is even, every function $f: X \rightarrow X / G$ homotopic to $\pi$ has a coincidence with $\pi$. If $k$ is odd and $G$ has an element which reverses orientation, then every function $f: X \rightarrow X / G$ homotopic to $\pi$ has a coincidence with $\pi$.

For, if we denote the degree of $g_{i} \in G$ by $a_{i}= \pm 1$, we have

$$
\mathscr{L}\left(g_{i}\right)=1+(-1)^{k} a_{i} \geqq 0 .
$$

If $k$ is even, $\mathscr{L}(e)=2$ for the identity element $e$ of the group and $\sum_{i=1}^{n} \mathfrak{L}\left(g_{i}\right) \neq 0$. If $k$ is odd and $G$ has an orientation reversing element $g_{j}$, then $a_{j}=-1$ and $\mathscr{L}\left(g_{j}\right)=2$. Hence $\sum_{l=1}^{n} \mathfrak{L}\left(g_{i}\right) \neq 0$.

\section{BiBLIOGRAPHY}

1. S. Eilenberg and N. Steenrod, Foundations of algebraic topology, Princeton University Press, 1952.

2. S. Lefschetz, Algebraic topology, Amer. Math. Soc. Colloquium Publications, vol. 27, 1942.

UNIVERSITY OF MiChIGAN AND

UNIVERSITY OF ILLINOIS 